\title{
Localisation uniforme des espaces de Besov et de Lizorkin-Triebel
}

\author{
Salah Eddine Allaoui et Gérard Bourdaud
}

29 septembre 2018

\begin{abstract}
Résumé
On établit des caractérisations intrinsèques des versions localisées-uniformes des espaces de Besov $B_{p, q}^{s}\left(\mathbb{R}^{n}\right)$, avec $p, q \in[1,+\infty]$, et de Lizorkin-Triebel $F_{p, q}^{s}\left(\mathbb{R}^{n}\right)$ avec $q \in[1,+\infty]$ et $p \in[1,+\infty[$, quel que soit le nombre réel $s>0$.
\end{abstract}

We give intrinsic characterisations for the uniformly localized versions of the Besov spaces $B_{p, q}^{s}\left(\mathbb{R}^{n}\right)$, where $p, q \in[1,+\infty]$, and of the Lizorkin-Triebel spaces $F_{p, q}^{s}\left(\mathbb{R}^{n}\right)$, where $q \in[1,+\infty]$ and $p \in[1,+\infty[$, whatever be the real number $s>0$.

Mots-clés : Espaces de Besov, Espaces de Lizorkin-Triebel, Localisation uniforme. 2010 Mathematics Subject Classification : 46E35.

\section{Introduction}

À tout espace normé $E$ de fonctions sur $\mathbb{R}^{n}$, il est possible d'associer sa version localiséeuniforme. Il s'agit de l'espace, noté $E_{l u}$, des fonctions $f$ telles que

$$
\sup _{a \in \mathbb{R}^{n}}\left\|\left(\tau_{a} \varphi\right) f\right\|_{E}<+\infty .
$$

Ici $\tau_{a}$ désigne l'opérateur de translation, défini par $\tau_{a} f(x):=f(x-a)$, et $\varphi$ est une fonction $C^{\infty}$ à support compact, positive, non nulle. Sous des hypothèses standard, rappelées au paragraphe 2, on peut montrer que $E_{l u}$ ne dépend aucunement du choix de la fonction auxiliaire $\varphi$.

Les espaces localisés-uniformes jouent un rôle dans diverses questions d'analyse mathématique. Par exemple, si $E$ est une algèbre de Banach de fonctions, pour la multiplication usuelle, il est naturel de conjecturer que l'ensemble des multiplicateurs de $E$ est précisément $E_{l u}$ - conjecture confirmée dans le cas des espaces de Sobolev $H^{s}\left(\mathbb{R}^{n}\right)$ pour $s>n / 2$, voir [4, p. 58] et [5, p. 151]. Ils interviennent aussi dans la caractérisation des fonctions qui opèrent, par composition à gauche, sur certains espaces de fonctions. Ainsi, les fonctions de $\mathbb{R}$ dans $\mathbb{R}$ qui opèrent, en ce sens, sur l'espace de Sobolev critique $W_{p}^{m}\left(\mathbb{R}^{n}\right)$, où l'entier $m$ vérifie $m=n / p>1$, sont précisément celles dont les dérivées appartiennent localement-uniformément à $W_{p}^{m-1}(\mathbb{R})[3$. L'extension de ce théorème aux espaces de Sobolev fractionnaires est une question ouverte. Cependant on a pu établir un résultat partiel [1] : si une fonction opère sur l'espace de Besov $B_{p, q}^{s}\left(\mathbb{R}^{n}\right)$, avec $s=n / p>1$ 
et $q>1$, alors sa dérivée appartient localement-uniformément à $B_{p, q}^{s-1}(\mathbb{R})$.

Il semble dès lors pertinent de décrire les localisations uniformes des espaces de Sobolev fractionnaires de façon intrinsèque, c'est-à-dire sans recourir à une fonction auxiliaire telle que la fonction $\varphi$ utilisée dans (11). À cet égard, rappelons que, pour les espaces $L_{p}\left(\mathbb{R}^{n}\right)_{l u}$, une telle description est bien connue, voir la proposition 3. C'est ce type de description, à l'aide d'intégrales portant sur les translatées d'une boule fixe, que nous mettrons en évidence pour les localisations uniformes des espaces de Sobolev fractionnaires.

\section{Plan}

La section 2 sera dévolue à des généralités sur la localisation uniforme. Dans la section 3 , on rappellera les définitions des espaces Besov et de Lizorkin-Triebel et on énoncera les théorèmes principaux, qui seront établis dans la dernière section.

\section{Notations et rappels}

La norme d'une fonction $f$ dans l'espace de Lebesgue $L_{p}\left(\mathbb{R}^{n}\right)$ est notée $\|f\|_{p}$. Comme d'habitude $c, c_{1}, \ldots$ désignera une constante positive pouvant dépendre de $n, s, p, q$ et des fonctions auxiliaires; sa valeur pourra changer d'une occurrence à l'autre. Rappelons une inégalité classique (voir, par exemple, [4, II.20, p. 44]) :

Lemme 1 Pour tout $q \in[1,+\infty[$ et tout réel $\alpha$, il existe $c>0$ tel que

$$
\sup _{0<t \leq 1 / 2} t^{\alpha} u(t) \leq c\left(\int_{0}^{1}\left(t^{\alpha} u(t)\right)^{q} \frac{\mathrm{d} t}{t}\right)^{1 / q}
$$

pour toute fonction croissante u sur l'intervalle ]0,1].

\section{Généralités sur la localisation uniforme}

Un espace de Banach de distributions (E.B.D.) sur $\mathbb{R}^{n}$ est un sous-espace vectoriel $E$ de $\mathcal{D}^{\prime}\left(\mathbb{R}^{n}\right)$ muni d'une norme complète $\|-\|_{E}$ telle que l'injection canonique $E \hookrightarrow \mathcal{D}^{\prime}\left(\mathbb{R}^{n}\right)$ soit continue. On dit que l'espace $E$ est un $\mathcal{D}\left(\mathbb{R}^{n}\right)$-module si $\phi f \in E$ pour tout $\phi \in \mathcal{D}\left(\mathbb{R}^{n}\right)$ et tout $f \in E$. Un E.B.D. E est isométriquement invariant par translation si $\tau_{a} f \in E$ et $\left\|\tau_{a} f\right\|_{E}=\|f\|_{E}$ pour tout $f \in E$ et tout $a \in \mathbb{R}^{n}$.

Proposition 1 Soit $E$ un $\mathcal{D}\left(\mathbb{R}^{n}\right)$-module isométriquement invariant par translation. Pour toute distribution $f$, les deux propriétés suivantes sont équivalentes :

(i) Il existe une fonction positive non nulle $\varphi \in \mathcal{D}\left(\mathbb{R}^{n}\right)$ vérifiant (11).

(ii) Pour toute fonction $\varphi \in \mathcal{D}\left(\mathbb{R}^{n}\right)$, on a la propriété (1).

Preuve. Voir [4, p. 57].

Si une distribution $f$ satisfait l'une des deux conditions équivalentes de la proposition 1, on dit que $f$ appartient localement uniformément à $E$; l'ensemble de ces distributions est 
noté $E_{l u}$. Soit une fonction positive non nulle $\varphi \in \mathcal{D}\left(\mathbb{R}^{n}\right)$. On montre facilement que $E_{l u}$ est un E.B.D. pour la norme

$$
\|f\|_{E_{l u}}:=\sup _{a \in \mathbb{R}^{n}}\left\|\left(\tau_{a} \varphi\right) f\right\|_{E}
$$

De la preuve de la proposition [1, il résulte qu'à équivalence près, la norme de $E_{l u}$ ne dépend pas du choix de la fonction $\varphi$.

Si $E$ est un E.B.D. et $m$ un entier positif, on peut considérer l'espace de Sobolev $W^{m}(E)$ d'ordre $m$ de base $E$, à savoir

$$
W^{m}(E):=\left\{f \in \mathcal{D}^{\prime}\left(\mathbb{R}^{n}\right): f^{(\alpha)} \in E \quad \text { pour tout } \quad|\alpha| \leq m\right\}
$$

$W^{m}(E)$ est un E.B.D. pour la norme

$$
\|f\|_{W^{m}(E)}:=\sum_{|\alpha| \leq m}\left\|f^{(\alpha)}\right\|_{E} .
$$

Proposition 2 Si E est un $\mathcal{D}\left(\mathbb{R}^{n}\right)$-module, isométriquement invariant par translation, il en est de même pour $W^{m}(E)$ et on a

$$
\left(W^{m}(E)\right)_{l u}=W^{m}\left(E_{l u}\right)
$$

avec des normes équivalentes.

Preuve. Elle résulte aisément de la formule de Leibniz et de la proposition 1 .

Nous terminerons cette section en rappelant la description de $L_{p}\left(\mathbb{R}^{n}\right)_{l u}$. La preuve facile est laissée au lecteur.

Proposition 3 Soit $p \in\left[1,+\infty\left[\right.\right.$. Soit $\mathbb{B}$ une boule ouverte (ou un cube ouvert) dans $\mathbb{R}^{n}$. Alors une fonction mesurable $f$ sur $\mathbb{R}^{n}$ appartient $\grave{a} L_{p}\left(\mathbb{R}^{n}\right)_{\text {lu }}$ si et seulement si

$$
\sup _{a \in \mathbb{R}^{n}}\left(\int_{\mathbb{B}+a}|f(x)|^{p} \mathrm{~d} x\right)^{1 / p}<+\infty ;
$$

de plus l'expression ci-dessus est équivalente à la norme $\|f\|_{L_{p}\left(\mathbb{R}^{n}\right)_{l u}}$.

\section{Définitions des espaces fonctionnels et énoncés des théorèmes}

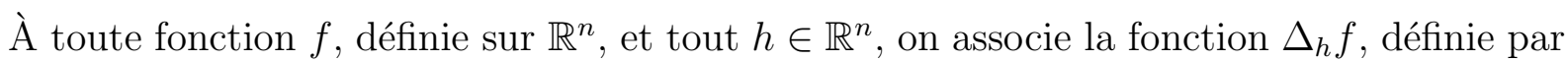
$\Delta_{h} f:=\tau_{-h} f-f$. Pour tout $p \in[1,+\infty]$, tout ensemble borélien $A$ de $\mathbb{R}^{n}$, toute fonction mesurable $f$ sur $\mathbb{R}^{n}$ et tout $t>0$, on pose

$$
\omega_{p, A}(f, t):=\sup _{|h| \leq t}\left(\int_{A}\left|\Delta_{h} f(x)\right|^{p} \mathrm{~d} x\right)^{1 / p}
$$




$$
\eta_{p, A}(f, t):=\sup _{|h| \leq t}\left(\int_{A}\left|\Delta_{h}^{2} f(x)\right|^{p} \mathrm{~d} x\right)^{1 / p} .
$$

On note simplement $\omega_{p}:=\omega_{p, \mathbb{R}^{n}}$, de même pour $\eta$.

Définition 1 Soient $0<s<1, p, q \in[1,+\infty]$. L'espace $B_{p, q}^{s}\left(\mathbb{R}^{n}\right)$ est l'ensemble des fonctions $f$ vérifiant

$$
\|f\|_{B_{p, q}^{s}\left(\mathbb{R}^{n}\right)}:=\|f\|_{p}+\left(\int_{0}^{1}\left(t^{-s} \omega_{p}(f, t)\right)^{q} \frac{\mathrm{d} t}{t}\right)^{1 / q}<+\infty .
$$

Définition 2 Soient $0<s<1, q \in[1,+\infty], 1 \leq p<\infty$. L'espace de Lizorkin-Triebel $F_{p, q}^{s}\left(\mathbb{R}^{n}\right)$ est l'ensemble des fonctions $f$ vérifiant

$$
\|f\|_{F_{p, q}^{s}\left(\mathbb{R}^{n}\right)}:=\|f\|_{p}+\left(\left(\int_{0}^{1}\left(t^{-s-n} \int_{|h| \leq t}\left|\Delta_{h} f(x)\right|^{q} \mathrm{~d} h\right)^{q} \frac{\mathrm{d} t}{t}\right)^{p / q} \mathrm{~d} x\right)^{1 / p}<+\infty .
$$

Rappelons qu'on obtient les mêmes espaces fonctionnels, avec des normes équivalentes, en remplaçant, dans les définitions précédentes, l'intégrale $\int_{0}^{1}$ par l'intégrale $\int_{0}^{r}$, où $r$ est n’importe quel réel positif fixé. Quand il n'y a pas lieu de distinguer entre les deux types d'espaces, $B$ ou $F$, nous posons $E_{p, q}^{s}\left(\mathbb{R}^{n}\right):=B_{p, q}^{s}\left(\mathbb{R}^{n}\right)$ ou $F_{p, q}^{s}\left(\mathbb{R}^{n}\right)$. Les espaces d'ordre 1 , c'est-à-dire les espaces $E_{p, q}^{1}\left(\mathbb{R}^{n}\right)$, se définissent de la même façon, à condition de remplacer $\Delta_{h}$ par l'opérateur de différence seconde $\Delta_{h}^{2}$ (et donc, dans le cas Besov, $\omega$ par $\eta$ ). Les espaces d'ordre supérieur à 1 sont, par définition, les espaces de Sobolev basés sur les espaces d'ordres compris entre 0 et 1 :

Définition 3 Soient $s>1$ et $m$ l'entier tel que $m<s \leq m+1$. Alors $E_{p, q}^{s}\left(\mathbb{R}^{n}\right)$ est l'ensemble des fonctions $f$ telles que $f^{(\alpha)} \in E_{p, q}^{s-m}\left(\mathbb{R}^{n}\right)$ pour tout $|\alpha| \leq m$. Cet espace est muni de la norme

$$
\sum_{|\alpha| \leq m}\left\|f^{(\alpha)}\right\|_{E_{p, q}^{s-m}\left(\mathbb{R}^{n}\right)}
$$

Venons-en à la description intrinsèque des espaces localisés-uniformes. On se limitera au cas $0<s \leq 1$, puisqu'il suffit d'appliquer la proposition 2 pour obtenir le cas général. Dans les énoncés suivants, $\mathbb{B}$ désignera une boule (ou un cube) fixé de $\mathbb{R}^{n}$. On supposera $p, q \in[1,+\infty](p<\infty$ dans le cas Lizorkin-Triebel).

Théorème 1 Si $0<s<1$, alors $B_{p, q}^{s}\left(\mathbb{R}^{n}\right)_{\text {lu }}$ est l'ensemble des fonctions $f$ telles que

$$
\sup _{a \in \mathbb{R}^{n}}\left(\int_{0}^{1}\left(t^{-s} \omega_{p, \mathbb{B}+a}(f, t)\right)^{q} \frac{\mathrm{d} t}{t}\right)^{1 / q}+\|f\|_{L_{p}\left(\mathbb{R}^{n}\right)_{l u}}<+\infty .
$$

De plus l'expression ci-dessus est une norme équivalente sur $B_{p, q}^{s}\left(\mathbb{R}^{n}\right)_{l u}$.

Théorème $2 B_{p, q}^{1}\left(\mathbb{R}^{n}\right)_{l u}$ est l'ensemble des fonctions $f$ telles que

$$
\sup _{a \in \mathbb{R}^{n}}\left(\int_{0}^{1}\left(t^{-1} \eta_{p, \mathbb{B}+a}(f, t)\right)^{q} \frac{\mathrm{d} t}{t}\right)^{1 / q}+\|f\|_{L_{p}\left(\mathbb{R}^{n}\right)_{l u}}<+\infty .
$$

De plus l'expression ci-dessus est une norme équivalente sur $B_{p, q}^{1}\left(\mathbb{R}^{n}\right)_{l u}$. 
Théorème 3 Si $0<s<1$, alors $F_{p, q}^{s}\left(\mathbb{R}^{n}\right)_{l u}$ est l'ensemble des fonctions $f$ telles que

$$
\sup _{a \in \mathbb{R}^{n}}\left\|\left(\int_{0}^{1}\left(t^{-s-n} \int_{|h| \leq t}\left|\Delta_{h} f(.)\right| \mathrm{d} h\right)^{q} \frac{\mathrm{d} t}{t}\right)^{1 / q}\right\|_{L_{p}(\mathbb{B}+a)}+\|f\|_{L_{p}\left(\mathbb{R}^{n}\right)_{l u}}<+\infty .
$$

De plus l'expression ci-dessus est une norme équivalente sur $F_{p, q}^{s}\left(\mathbb{R}^{n}\right)_{l u}$.

Théorème $4 F_{p, q}^{1}\left(\mathbb{R}^{n}\right)_{l u}$ est l'ensemble des fonctions $f$ telles que

$$
\sup _{a \in \mathbb{R}^{n}}\left\|\left(\int_{0}^{1}\left(t^{-n-1} \int_{|h| \leq t}\left|\Delta_{h}^{2} f(.)\right| \mathrm{d} h\right)^{q} \frac{\mathrm{d} t}{t}\right)^{1 / q}\right\|_{L_{p}(\mathbb{B}+a)}+\|f\|_{L_{p}\left(\mathbb{R}^{n}\right) l u}<+\infty .
$$

De plus l'expression ci-dessus est une norme équivalente sur $F_{p, q}^{1}\left(\mathbb{R}^{n}\right)_{l u}$.

\section{Preuves des théorèmes}

Sans perte de généralité, on peut supposer que $\mathbb{B}$ est la boule unité de $\mathbb{R}^{n}$. Dans cette section, on fixe deux fonctions $\varphi_{0}$ et $\varphi_{1}$ dans $\mathcal{D}\left(\mathbb{R}^{n}\right)$, telles que :

- $0 \leq \varphi_{0} \leq 1, \varphi_{0}$ est non nulle et portée par $\mathbb{B} / 4$,

$-\varphi_{1}(x)=1$ sur $4 \mathbb{B}$.

\subsection{Preuve du théorème 1}

On utilisera la formule suivante, valable pour tout $h \in \mathbb{R}^{n}$ et toutes fonctions $f$ et $g$ sur $\mathbb{R}^{n}$ :

$$
\Delta_{h}(f g)=\left(\Delta_{h} f\right)\left(\tau_{-h} g\right)+f\left(\Delta_{h} g\right) .
$$

Désignons par $A(f)$ le premier terme de l'inégalité (2) .

\subsection{1 Étape 1}

Soit $f$ une fonction telle que $A(f)<\infty$. Par la formule (6), on a, pour tous $a, h \in \mathbb{R}^{n}$ et $|h| \leq t \leq 1 / 2$

$$
\begin{gathered}
\left(\int_{\mathbb{R}^{n}}\left|\Delta_{h}\left(\left(\tau_{a} \varphi_{0}\right) f\right)(x)\right|^{p} \mathrm{~d} x\right)^{1 / p} \\
\leq\left(\int_{\mathbb{R}^{n}}\left|\Delta_{h} f(x) \varphi_{0}(x+h-a)\right|^{p} \mathrm{~d} x\right)^{1 / p}+\left(\int_{\mathbb{R}^{n}}|f(x)|^{p}\left|\Delta_{h}\left(\tau_{a} \varphi_{0}\right)(x)\right|^{p} \mathrm{~d} x\right)^{1 / p} \\
\leq\left(\int_{\mathbb{B}+a}\left|\Delta_{h} f(x)\right|^{p} \mathrm{~d} x\right)^{1 / p}+t\left\|\nabla \varphi_{0}\right\|_{\infty}\left(\int_{\mathbb{B}+a}|f(x)|^{p} \mathrm{~d} x\right)^{1 / p} \\
\leq c_{1}\left(\omega_{p, \mathbb{B}+a}(f, t)+t\|f\|_{L^{p}\left(\mathbb{R}^{n}\right)_{l u}}\right) .
\end{gathered}
$$

Par la condition $s<1$, on voit que

$$
\left(\int_{0}^{1 / 2}\left(t^{-s} \omega_{p}\left(\left(\tau_{a} \varphi_{0}\right) f, t\right)\right)^{q} \frac{\mathrm{d} t}{t}\right)^{1 / q}
$$




$$
\leq c_{1}\left(\left(\int_{0}^{1 / 2}\left(t^{-s} \omega_{p, \mathbb{B}+a}(f, t)\right)^{q} \frac{\mathrm{d} t}{t}\right)^{1 / q}+\left(\int_{0}^{1 / 2}\left(t^{1-s}\right)^{q} \frac{\mathrm{d} t}{t}\right)^{1 / q}\|f\|_{L^{p}\left(\mathbb{R}^{n}\right)_{l u}}\right) .
$$

L'expression ci-dessus étant majorée par $c_{2} A(f)$, pour une certaine constante $c_{2}$, il vient

$$
\sup _{a \in \mathbb{R}^{n}}\left\|\left(\tau_{a} \varphi_{0}\right) f\right\|_{B_{p, q}^{s}\left(\mathbb{R}^{n}\right)} \leq c_{3} A(f) .
$$

\subsection{2 Étape 2}

Soit $f \in B_{p, q}^{s}\left(\mathbb{R}^{n}\right)_{l u}$. On voit aussitôt que $\Delta_{h}\left(\left(\tau_{a} \varphi_{1}\right) f\right)(x)=\Delta_{h} f(x)$ pour tout $a \in \mathbb{R}^{n}$, tout $x \in \mathbb{B}+a$, et tout $|h| \leq 1$. On en déduit aisément que

$$
A(f) \leq c_{4} \sup _{a \in \mathbb{R}^{n}}\left\|\left(\tau_{a} \varphi_{1}\right) f\right\|_{F_{p, q}^{s}\left(\mathbb{R}^{n}\right)}
$$

\subsection{Preuve du théorème 2}

On désignera par $A(f)$ le premier terme de l'inégalité (3) et on posera

$$
M_{p, a}(f):=\sup _{0<t \leq 1 / 2} \frac{1}{t} \eta_{p, \mathbb{B}+a}(f, t) .
$$

\subsubsection{Résultats préliminaires}

On dispose des formules suivantes, où $k \in \mathbb{N}^{*}, h \in \mathbb{R}^{n}$ et où $f$ et $g$ sont des fonctions quelconques :

$$
\begin{gathered}
\Delta_{h}^{2}(f g)=\left(\Delta_{h}^{2} f\right)\left(\tau_{-2 h} g\right)+\left(\Delta_{h}^{2} g\right)\left(\tau_{-h} f\right)+\left(\Delta_{h} f\right)\left(\Delta_{2 h} g\right), \\
\Delta_{h}=2^{-k} \Delta_{2^{k} h}-\sum_{l=0}^{k-1} 2^{-l-1} \Delta_{2^{l} h}^{2} .
\end{gathered}
$$

La première est immédiate, la seconde s'obtient facilement par récurrence sur $k$.

Lemme 2 Il existe $c>0$ tel que

$$
\omega_{p, \mathbb{B}+a}(f, t) \leq c t\left\{\left(\int_{2 \mathbb{B}+a}|f(x)|^{p} \mathrm{~d} x\right)^{1 / p}+M_{p, a}(f)|\ln t|\right\}
$$

pour tout $0<t \leq 1 / 2$, tout $a \in \mathbb{R}^{n}$ et toute fonction localement intégrable $f$.

Preuve. Le lemme est une variante de l'inégalité classique de Marchaud. On définit l'entier $k \geq 1$ par l'encadrement $2^{-k-1}<t \leq 2^{-k}$. De la formule (8), on déduit, pour $|h| \leq t$

$$
\begin{gathered}
\left(\int_{\mathbb{B}+a}\left|\Delta_{h} f(x)\right|^{p} \mathrm{~d} x\right)^{1 / p} \\
\leq 2^{-k}\left(\int_{\mathbb{B}+a}\left|\Delta_{2^{k} h} f(x)\right|^{p} \mathrm{~d} x\right)^{1 / p}+\sum_{l=0}^{k-1} 2^{-l-1}\left(\int_{\mathbb{B}+a}\left|\Delta_{2^{l} h}^{2} f(x)\right|^{p} \mathrm{~d} x\right)^{1 / p}
\end{gathered}
$$




$$
\begin{gathered}
\leq 2^{-k+1}\left(\int_{2 \mathbb{B}+a}|f(x)|^{p} \mathrm{~d} x\right)^{1 / p}+\sum_{l=0}^{k-1} 2^{-l-1}\left(2^{l-k} M_{p, a}(f)\right), \\
\leq 4 t\left(\int_{2 \mathbb{B}+a}|f(x)|^{p} \mathrm{~d} x\right)^{1 / p}+\frac{1}{\ln 2} t|\ln t| M_{p, a}(f),
\end{gathered}
$$

ce qui conclut la preuve du lemme 2.

\subsection{2 Étape 1}

Soit $f$ une fonction telle que $A(f)<\infty$. Par la formule (7), il vient, pour $|h| \leq t \leq 1 / 4$,

$$
\begin{gathered}
\left(\int_{\mathbb{R}^{n}}\left|\Delta_{h}^{2}\left(\left(\tau_{a} \varphi_{0}\right) f\right)(x)\right|^{p} \mathrm{~d} x\right)^{1 / p} \leq\left(\int_{\mathbb{R}^{n}}\left|\Delta_{h}^{2} f(x) \varphi_{0}(x+2 h-a)\right|^{p} \mathrm{~d} x\right)^{1 / p} \\
+\left(\int_{\mathbb{R}^{n}}|f(x+h)|^{p}\left|\Delta_{h}^{2}\left(\tau_{a} \varphi_{0}\right)(x)\right|^{p} \mathrm{~d} x\right)^{1 / p} \\
+\left(\int_{\mathbb{R}^{n}}\left|\Delta_{h} f(x)\left(\varphi_{0}(x+2 h-a)-\varphi_{0}(x-a)\right)\right|^{p} \mathrm{~d} x\right)^{1 / p} \\
\leq\left(\int_{\mathbb{B}+a}\left|\Delta_{h}^{2} f(x)\right|^{p} \mathrm{~d} x\right)^{1 / p}+c_{1} t^{2}\left(\int_{\mathbb{B}+a}|f(x)|^{p} \mathrm{~d} x\right)^{1 / p}+c_{2} t\left(\int_{\mathbb{B}+a}\left|\Delta_{h} f(x)\right|^{p} \mathrm{~d} x\right)^{1 / p} \\
\leq c_{3}\left(\eta_{p, \mathbb{B}+a}(f, t)+t^{2}\|f\|_{L^{p}\left(\mathbb{R}^{n}\right)_{l u}}+t \omega_{p, \mathbb{B}+a}(f, t)\right),
\end{gathered}
$$

et donc

$$
\begin{gathered}
\left(\int_{0}^{1 / 4}\left(t^{-1} \eta_{p}\left(\left(\tau_{a} \varphi_{0}\right) f, t\right)\right)^{q} \frac{\mathrm{d} t}{t}\right)^{1 / q} \\
\leq c_{3}\left(\int_{0}^{1 / 4}\left(t^{-1} \eta_{p, \mathbb{B}+a}(f, t)\right)^{q} \frac{\mathrm{d} t}{t}\right)^{1 / q}+c_{3}\|f\|_{L^{p}\left(\mathbb{R}^{n}\right)_{l u}}\left(\int_{0}^{1 / 4} t^{q-1} \mathrm{~d} t\right)^{1 / q} \\
+c_{3}\left(\int_{0}^{1 / 4}\left(\omega_{p, \mathbb{B}+a}(f, t)\right)^{q} \frac{\mathrm{d} t}{t}\right)^{1 / q} .
\end{gathered}
$$

En conséquence

$$
\sup _{a \in \mathbb{R}^{n}}\left\|\left(\tau_{a} \varphi_{0}\right) f\right\|_{B_{p, q}^{1}\left(\mathbb{R}^{n}\right)} \leq c_{4}\left(A(f)+\sup _{a \in \mathbb{R}^{n}}\left(\int_{0}^{1 / 4}\left(\omega_{p, \mathbb{B}+a}(f, t)\right)^{q} \frac{\mathrm{d} t}{t}\right)^{1 / q}\right) .
$$

Grâce au lemme 2, on a, pour tout $a \in \mathbb{R}^{n}$,

$$
\begin{gathered}
\left(\int_{0}^{1 / 4}\left(\omega_{p, \mathbb{B}+a}(f, t)\right)^{q} \frac{\mathrm{d} t}{t}\right)^{1 / q} \\
\leq c_{5}\|f\|_{L^{p}\left(\mathbb{R}^{n}\right)_{l u}}\left(\int_{0}^{1 / 4} t^{q-1} \mathrm{~d} t\right)^{1 / q}+c_{6} M_{p, a}(f)\left(\int_{0}^{1 / 4} t^{q-1}|\ln t|^{q} \mathrm{~d} t\right)^{1 / q} .
\end{gathered}
$$

En appliquant le lemme 1 à la fonction croissante $t \mapsto \eta_{p, \mathbb{B}+a}(f, t)$, on conclut que

$$
\sup _{a \in \mathbb{R}^{n}}\left\|\left(\tau_{a} \varphi_{0}\right) f\right\|_{B_{p, q}^{1}\left(\mathbb{R}^{n}\right)} \leq c_{7} A(f)
$$




\subsection{3 Étape 2}

Soit $f \in B_{p, q}^{1}\left(\mathbb{R}^{n}\right)_{l u}$. En procédant comme dans l'étape 2 de la preuve du théorème $\mathbb{1}$, il vient

$$
A(f) \leq c_{8}\|f\|_{B_{p, q}^{1}\left(\mathbb{R}^{n}\right)_{l u}} .
$$

\subsection{Preuve du théorème 3}

On désignera par $A(f)$ le premier terme de l'inégalité (44).

\subsection{1 Étape 1}

Soit $f$ une fonction telle que $A(f)<\infty$. Par la formule (6), nous avons

$$
\begin{gathered}
\int_{|h| \leq t}\left|\Delta_{h}\left(\left(\tau_{a} \varphi_{0}\right) f\right)(x)\right| \mathrm{d} h \\
\leq \int_{|h| \leq t}\left|\Delta_{h} f(x)\right| \varphi_{0}(x+h-a) \mathrm{d} h+|f(x)| \int_{|h| \leq t}\left|\Delta_{h}\left(\tau_{a} \varphi_{0}\right)(x)\right| \mathrm{d} h .
\end{gathered}
$$

On obtient

$$
\begin{gathered}
\left(\int_{\mathbb{R}^{n}}\left(\int_{0}^{1 / 2}\left(t^{-s-n} \int_{|h| \leq t}\left|\Delta_{h}\left(\left(\tau_{a} \varphi_{0}\right) f\right)(x)\right| \mathrm{d} h\right)^{q} \frac{\mathrm{d} t}{t}\right)^{p / q} \mathrm{~d} x\right)^{1 / p} \\
\leq\left(\int_{\mathbb{B}+a}\left(\int_{0}^{1 / 2}\left(t^{-s-n} \int_{|h| \leq t}\left|\Delta_{h} f(x)\right| \mathrm{d} h\right)^{q} \frac{\mathrm{d} t}{t}\right)^{p / q} \mathrm{~d} x\right)^{1 / p}+c_{1}\left(\int_{\mathbb{B}+a}|f(x)|^{p} \mathrm{~d} x\right)^{1 / p},
\end{gathered}
$$

ce qui nous donne

$$
\sup _{a \in \mathbb{R}^{n}}\left\|\left(\tau_{a} \varphi_{0}\right) f\right\|_{F_{p, q}^{s}\left(\mathbb{R}^{n}\right)} \leq c_{2} A(f) .
$$

\subsection{2 Étape 2}

Supposons que $f \in F_{p, q}^{s}\left(\mathbb{R}^{n}\right)_{l u}$. En procédant comme dans l'étape 2 de la preuve du théorème 1, il vient

$$
A(f) \leq c_{3}\|f\|_{F_{p, q}^{s}\left(\mathbb{R}^{n}\right)_{l u}} .
$$

\subsection{Preuve du théorème 4}

On désignera par $A(f)$ le premier terme de l'inégalité (5).

\subsection{1 Étape 1}

Soit $f$ une fonction telle que $A(f)<\infty$. Soit

$$
G(x):=\left(\int_{0}^{1}\left(t^{-n-1} \int_{|h| \leq t}\left|\Delta_{h}^{2} f(x)\right| \mathrm{d} h\right)^{q} \frac{\mathrm{d} t}{t}\right)^{1 / q} .
$$


Par la formule (7), il vient, pour tous $a, x \in \mathbb{R}^{n}$ et $t>0$,

$$
\begin{gathered}
\int_{|h| \leq t}\left|\Delta_{h}^{2}\left(\left(\tau_{a} \varphi_{0}\right) f\right)(x)\right| \mathrm{d} h \\
\leq \int_{|h| \leq t}\left|\Delta_{h}^{2} f(x)\right| \varphi_{0}(x+2 h-a) \mathrm{d} h+\int_{|h| \leq t}|f(x+h)|\left|\Delta_{h}^{2}\left(\tau_{a} \varphi_{0}\right)(x)\right| \mathrm{d} h \\
+\int_{|h| \leq t}\left|\Delta_{h} f(x)\right|\left|\Delta_{2 h} \tau_{a} \varphi_{0}(x)\right| \mathrm{d} h .
\end{gathered}
$$

On en déduit, pour tout $a \in \mathbb{R}^{n}$,

$$
\begin{gathered}
\left(\int_{\mathbb{R}^{n}}\left(\int_{0}^{1 / 16}\left(t^{-n-1} \int_{|h| \leq t}\left|\Delta_{h}^{2}\left(\left(\tau_{a} \varphi_{0}\right) f\right)(x)\right| \mathrm{d} h\right)^{q} \frac{\mathrm{d} t}{t}\right)^{p / q} \mathrm{~d} x\right)^{1 / p} \\
\leq\left(\int_{\frac{\mathbb{B}}{2}+a}\left(\int_{0}^{1 / 16}\left(t^{-n-1} \int_{|h| \leq t}\left|\Delta_{h}^{2}\left(\left(\tau_{a} \varphi_{0}\right) f\right)(x)\right| \mathrm{d} h\right)^{q} \frac{\mathrm{d} t}{t}\right)^{p / q} \mathrm{~d} x\right)^{1 / p} \\
\leq c_{1}(U(a)+V(a)+W(a)),
\end{gathered}
$$

où

$$
\begin{aligned}
U(a) & :=\left(\int_{\frac{\mathbb{B}}{2}+a}\left(\int_{0}^{1 / 16}\left(t^{-n-1} \int_{|h| \leq t}\left|\Delta_{h}^{2} f(x)\right| \mathrm{d} h\right)^{q} \frac{\mathrm{d} t}{t}\right)^{p / q} \mathrm{~d} x\right)^{1 / p} \\
V(a) & :=\left(\int_{\frac{\mathbb{B}}{2}+a}\left(\int_{0}^{1 / 16}\left(t^{-n+1} \int_{|h| \leq t}|f(x+h)| \mathrm{d} h\right)^{q} \frac{\mathrm{d} t}{t}\right)^{p / q} \mathrm{~d} x\right)^{1 / p}, \\
W(a) & :=\left(\int_{\frac{\mathbb{B}}{2}+a}\left(\int_{0}^{1 / 16}\left(t^{-n} \int_{|h| \leq t}\left|\Delta_{h} f(x)\right| \mathrm{d} h\right)^{q} \frac{\mathrm{d} t}{t}\right)^{p / q} \mathrm{~d} x\right)^{1 / p} .
\end{aligned}
$$

On voit facilement que

$$
U(a)+V(a) \leq c_{2}\left(\int_{\mathbb{B}+a} G(x)^{p} \mathrm{~d} x\right)^{1 / p}+c_{3}\|f\|_{L_{p}(\mathbb{B}+a)} .
$$

Estimation de $W(a)$. Posons

$$
G_{1}(x):=\left(\int_{0}^{1 / 16}\left(t^{-n} \int_{|h| \leq t}\left|\Delta_{h} f(x)\right| \mathrm{d} h\right)^{q} \frac{\mathrm{d} t}{t}\right)^{1 / q} .
$$

En décomposant l'intervalle ]0,1/16] en intervalles dyadiques et en utilisant des majorations évi-dentes, on obtient $G_{1}(x) \leq c_{4} G_{2}(x)$, où

$$
G_{2}(x):=\left(\sum_{j \geq 4}\left(2^{j n} \int_{|h| \leq 2^{-j}}\left|\Delta_{h} f(x)\right| \mathrm{d} h\right)^{q}\right)^{1 / q} .
$$


Par le changement de variable $h^{\prime}=2^{j-3} h$, il vient

$$
G_{2}(x)=\left(\sum_{j \geq 4}\left(\int_{|h| \leq 1 / 8}\left|\Delta_{2^{-j+3} h} f(x)\right| \mathrm{d} h\right)^{q}\right)^{1 / q} .
$$

Par (8), on a

$$
\Delta_{2^{-j+3} h}=2^{-j+3} \Delta_{h}-\sum_{\ell=0}^{j-4} 2^{-l-1} \Delta_{2^{\ell-j+3} h}^{2},
$$

d'où $G_{2}(x) \leq c_{5}\left(G_{3}(x)+G_{4}(x)\right)$, avec

$$
G_{3}(x):=\left(\sum_{j \geq 4}\left(2^{-j} \int_{|h| \leq 1 / 8}\left|\Delta_{h} f(x)\right| \mathrm{d} h\right)^{q}\right)^{1 / q},
$$

et

$$
G_{4}(x):=\left(\sum_{j \geq 4}\left(\int_{|h| \leq 1 / 8} \sum_{\ell=0}^{j-4} 2^{-\ell-1}\left|\Delta_{2^{\ell-j+3} h}^{2} f(x)\right| \mathrm{d} h\right)^{q}\right)^{1 / q} .
$$

Estimation de $G_{3}$. On a aussitôt

$$
G_{3}(x)=c_{6} \int_{|h| \leq 1 / 8}\left|\Delta_{h} f(x)\right| \mathrm{d} h .
$$

L'inégalité de Minkowski nous donne, pour tout $a \in \mathbb{R}^{n}$,

$$
\begin{gathered}
\left(\int_{\frac{\mathbb{B}}{2}+a} G_{3}(x)^{p} \mathrm{~d} x\right)^{1 / p} \leq c_{6} \int_{|h| \leq 1 / 8}\left\{\int_{\frac{\mathbb{B}}{2}+a}\left|\Delta_{h} f(x)\right|^{p} \mathrm{~d} x\right\}^{1 / p} \mathrm{~d} h \\
\leq c_{6} \int_{|h| \leq 1 / 8}\left\{\int_{\frac{\mathbb{B}}{2}+a}|f(x+h)|^{p} \mathrm{~d} x\right\}^{1 / p} \mathrm{~d} h+c_{7}\left(\int_{\frac{\mathbb{B}}{2}+a}|f(x)|^{p} \mathrm{~d} x\right)^{1 / p} \\
\leq c_{6} \int_{|h| \leq 1 / 8}\left\{\int_{\mathbb{B}+a}|f(x)|^{p} \mathrm{~d} x\right\}^{1 / p} \mathrm{~d} h+c_{7}\left(\int_{\frac{\mathbb{B}}{2}+a}|f(x)|^{p} \mathrm{~d} x\right)^{1 / p} \\
\leq c_{8}\left(\int_{\mathbb{B}+a}|f(x)|^{p} \mathrm{~d} x\right)^{1 / p} .
\end{gathered}
$$

Estimation de $G_{4}$. Par le lemme 1, on a, pour tout $x \in \mathbb{R}^{n}$ et $0<t \leq 1 / 2$,

$$
\int_{|h| \leq t}\left|\Delta_{h}^{2} f(x)\right| \mathrm{d} h \leq c_{9} t^{n+1} G(x) .
$$

En raison du plongement $\ell_{1} \hookrightarrow \ell_{q}$, on a

$$
G_{4}(x) \leq \sum_{j \geq 4} \int_{|h| \leq 1 / 8} \sum_{\ell=0}^{j-4} 2^{-\ell-1}\left|\Delta_{2^{\ell-j+3} h}^{2} f(x)\right| \mathrm{d} h .
$$


On vérifie facilement que

$$
\int_{|h| \leq 1 / 8}\left|\Delta_{2^{\ell-j+3} h}^{2} f(x)\right| \mathrm{d} h=2^{-3 n} 2^{(j-\ell) n} \int_{|h| \leq 2^{\ell-j}}\left|\Delta_{h}^{2} f(x)\right| \mathrm{d} h .
$$

En combinant cette relation avec l'inégalité (10), on obtient

$$
G_{4}(x) \leq c_{10} G(x) \sum_{j \geq 4} \sum_{\ell=0}^{j-4} 2^{-\ell-1} 2^{\ell-j}=c_{11} G(x) .
$$

Il vient donc, pour tout $a \in \mathbb{R}^{n}$,

$$
\left(\int_{\frac{\mathbb{B}}{2}+a} G_{4}(x)^{p} \mathrm{~d} x\right)^{1 / p} \leq c_{11}\left(\int_{\mathbb{B}+a} G(x)^{p} \mathrm{~d} x\right)^{1 / p} .
$$

En tenant compte des estimations obtenues pour $G_{3}$ et $G_{4}$, on peut conclure que l'expression $W(a)$ est estimée par

$$
\left(\int_{\mathbb{B}+a} G(x)^{p} \mathrm{~d} x\right)^{1 / p}+\left(\int_{\mathbb{B}+a}|f(x)|^{p} \mathrm{~d} x\right)^{1 / p} .
$$

En combinant avec (9), on conclut que

$$
\sup _{a \in \mathbb{R}^{n}}\left\|\left(\tau_{a} \varphi_{0}\right) f\right\|_{F_{p, q}^{1}\left(\mathbb{R}^{n}\right)} \leq c_{12} A(f)
$$

\subsection{2 Étape 2}

Supposons que $f \in F_{p, q}^{1}\left(\mathbb{R}^{n}\right)_{l u}$. En procédant comme dans l'étape 2 de la preuve du théorème 1, il vient

$$
A(f) \leq c_{13}\|f\|_{F_{p, q}^{1}\left(\mathbb{R}^{n}\right)_{l u}}
$$

\section{Références}

[1] S.E. Allaoui, G. Bourdaud. Composition dans les espaces de Besov critiques. Ann. Fac. Sci. Toulouse, Math. 25 (2016), 875-893.

[2] G. Bourdaud. Localisations des espaces de Besov. Studia Math. 90 (1988), 153-163.

[3] G. Bourdaud. Le calcul fonctionnel dans les espaces de Sobolev. Invent. Math. 104 (1991), 435-446.

[4] G. Bourdaud. Analyse fonctionnelle dans l'espace Euclidien, 2ème édition, Pub. Math. Univ. Paris 7, 23 (1995).

[5] J. Peetre. New thoughts on Besov spaces. Duke Univ. Math. Series I, Durham, N.C., 1976. 
Salah Eddine Allaoui

Département de Mathématique et Informatique

Université de Laghouat

Laghouat 03000

Algérie

shallaoui@yahoo.fr

Gérard Bourdaud

Université Paris Diderot, I.M.J. - P.R.G (UMR 7586)

Bâtiment Sophie Germain

Case 7012

75205 Paris Cedex 13

bourdaud@math.univ-paris-diderot.fr 